\title{
Conductance Model for Single-Crystalline/Compact Metal Oxide Gas-Sensing Layers in the Nondegenerate Limit: Example of Epitaxial $\mathrm{SnO}_{2}(101)$
}

\author{
Cristian Eugen Simion, ${ }^{\dagger}$ Federico Schipani, ${ }^{\ddagger}$ Alexandra Papadogianni, ${ }^{\S}$ Adelina Stanoiu, ${ }^{\dagger}$ \\ Melanie Budde, ${ }^{\S}$ Alexandru Oprea, ${ }^{\ddagger}$ Udo Weimar,, Oliver Bierwagen, $^{\S}$ and Nicolae Barsan ${ }^{*} \neq \odot$ \\ ${ }^{\dagger}$ National Institute of Materials Physics, Atomistilor 405A, 077125 Magurele, Romania \\ ${ }^{\ddagger}$ Institute of Physical and Theoretical Chemistry, University of Tübingen, Auf der Morgenstelle 15, 72076 Tübingen, Germany \\ ${ }^{\S}$ Paul-Drude-Institut für Festkörperelektronik, Leibniz-Institut im Forschungsverbund Berlin e.V., Hausvogteiplatz 5-7, 10117 \\ Berlin, Germany
}

\section{Supporting Information}

\begin{abstract}
Semiconducting metal oxide (SMOX)-based gas sensors are indispensable for safety and health applications, for example, explosive, toxic gas alarms, controls for intake into car cabins, and monitor for industrial processes. In the past, the sensor community has been studying polycrystalline materials as sensors where the porous and random microstructure of the SMOX does not allow a separation of the phenomena involved in the sensing process. This led to conduction models that can model and predict the behavior of the overall response, but they were not capable of giving fundamental information regarding the basic mechanisms taking place. The study of epitaxial layers is a definite improvement, allowing clarifying the different aspects and contributions of the sensing mechanisms. A detailed analytical model of the transduction function for $\mathrm{n}$ - and $\mathrm{p}$-type single-crystalline/compact metal oxide gas sensors was developed that directly relates the conductance of the sample with changes in the surface electrostatic potential. Combined dc resistance and work function measurements were used in a compact $\mathrm{SnO}_{2}(101)$ layer in operando conditions that allowed us to check the validity of our model in the region where Boltzmann approximation holds to determine the surface and bulk properties of the material.

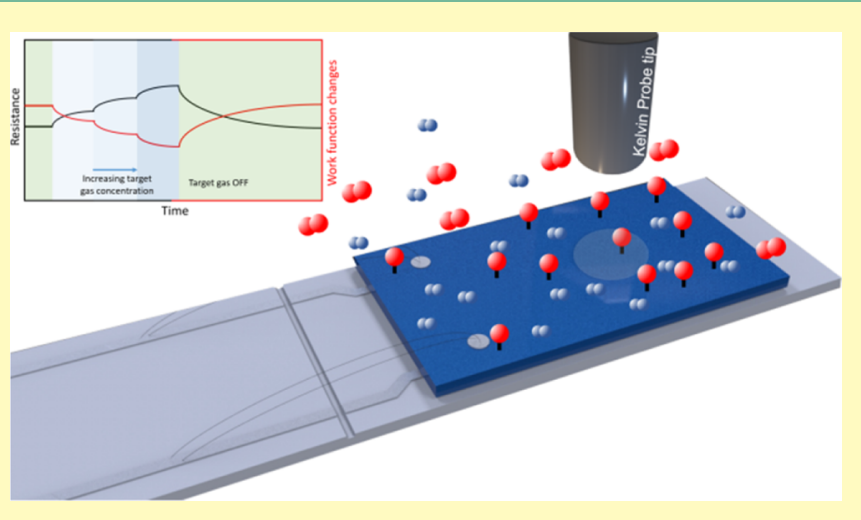

KEYWORDS: compact layers, epitaxial $\mathrm{SnO}_{2}$, single-crystalline, conduction model, SMOX, gas sensor

\section{INTRODUCTION}

More than 60 years ago, Heiland et al. ${ }^{1,2}$ showed that the conductivity of semiconducting metal oxides (SMOX) (using single-crystalline $\mathrm{ZnO}$ ) depends on the composition of the surrounding atmosphere. More than 50 years ago, Seiyama et $\mathrm{al}^{3}$ proposed the use of gas chromatographs equipped with SMOX-ZnO thin film-based detectors. The first gas sensors based on $\mathrm{SnO}_{2}$, for the detection of natural gas leakages, were realized by Taguchi, ${ }^{4}$ who later founded Figaro Eng., the first company to commercialize SMOX-based gas sensors in Japan. Since then, SMOX-based sensors have been used in various applications, for example, explosive and toxic gas alarms, controls for air intake into car cabins, monitors for industrial processes, and so forth. ${ }^{5,6}$ Their technological realization was significantly changed and the combination between thick porous sensing layers and micro-electro-mechanical system substrates, which offers significant miniaturization, cost reduction, and lowering of the power consumption is now the state of the art. ${ }^{7,8}$

Recent developments are integrating different sensing materials on the same chip together with driving and evaluation electronics, ${ }^{9}$ heralding the occurrence of IoT-ready SMOXbased sensing devices.

In principle, detection with SMOX-based devices is simple; one generally assumes that in air, at temperatures between 150 and $400{ }^{\circ} \mathrm{C}$, atmospheric oxygen adsorbs at the surface of the metal oxides by trapping electrons from the bulk. This results in an overall increase of the sensor's resistance, for n-type materials, or a decrease, for p-type materials. The presence of gases in the atmosphere that react with the preadsorbed oxygen or directly with the metal oxide also results in resistance changes (sensor signals). Accordingly, two aspects must be examined: the surface reaction taking place between the material and the gases

Received: June 3, 2019

Accepted: August 15, 2019

Published: August 15, 2019 
together with the associated electrical charge transfer processes (sometimes called the receptor function) and their translation into the corresponding changes in the electrical resistance of the sensing layer (the transduction function); ${ }^{10}$ the latter plays a very important role, for example, possibly making the difference between the performances of $\mathrm{p}$ - and n-type SMOX-based gas sensors. ${ }^{11}$ For understanding the transduction function, performing simultaneous work function and $\mathrm{dc}$ resistance measurements is extremely useful because it allows to directly link the change in the surface band bending with the sensor signals. $^{12}$ In combination with appropriate modeling, this investigation method was proven to be very effective. ${ }^{13-17}$ Most research effort, including modeling, was focused on porous sensing layers based on n-type SMOX because this combination is offering the best performance; the reason is that the transduction of the changes of the surface potential, band bending, is exponentially translated into changes of the electrical resistance of the sensing layer. ${ }^{18}$ Of late, there is a renewed interest for studying model systems, especially SMOX epitaxial layers. ${ }^{19,20,29}$ Such studies answer the need to clarify different aspects of the fundamental understanding of sensing that are not possible to clarify by studying polycrystalline samples. For example, by combining operando infrared studies and density functional theory calculations, it was found that the different ways in which water vapor influences the $\mathrm{CO}$ sensing of differently prepared $\mathrm{SnO}_{2}$-sensing materials can be attributed to the reaction taking place on different crystalline surfaces, namely, (110) and (101). ${ }^{21}$ Definitive proof can be provided only by studying the corresponding epitaxial layers.

Accordingly, here, we developed a detailed model for the relationship between the conductance of compact $n$ - and $p$-type SMOX single-crystalline films and the changes in the surface electrostatic potential determined by the charging of the surface with positive or negative charge. The model is restricted to cases in which the only charge carriers are electrons or holes that are obeying the Boltzmann statistics and there is no exchange of matter between the atmosphere and the bulk of the layer. In order to check its applicability, we realized and characterized epitaxial $\mathrm{SnO}_{2}$ layers. The results of the combined work function/dc resistance measurements were used as inputs for calculations based on the model and allowed for the determination of the surface and bulk properties of the material.

\section{THEORETICAL MODEL}

The total conductance of a compact layer that is having its surface exposed to the ambient atmosphere is the sum of the conductance of the part of the layer influenced by the surface phenomena (called surface layer) and the conductance of the layer that is left unchanged (called bulk); we will call those surface and volume contributions.

$$
G_{\text {total }}=G_{\mathrm{s}}+G_{\mathrm{b}}
$$

More in detail, eq 1 becomes

$$
G_{\text {total }}=\widetilde{\sigma}_{\mathrm{s}} \frac{z_{0} W}{L}+\sigma_{\mathrm{b}} \frac{\left(D-z_{0}\right) W}{L}
$$

where $L$ is the length of the layer, $W$ is its width, $D$ is its thickness, and $z_{0}$ is the thickness of the surface layer. $\widetilde{\sigma}_{\mathrm{s}}$ is the average conductivity of the surface layer and $\sigma_{\mathrm{b}}$ is the conductivity of the bulk. For a more thorough analysis, we will look to the possible specific cases. For the case of n-type SMOX, the calculations are presented in detail. Because of the similarities, for the case of $\mathrm{p}$ - type SMOX, only the final results are presented. In our calculations, we used inputs from the book of Morrison. ${ }^{22}$

n-Type SMOX - Surface Effects That Do Not Affect the Full Layer. For an n-type SMOX, using the definition for conductivity, $\widetilde{\sigma}_{\mathrm{s}}=e \mu \tilde{n}_{\mathrm{s}}$ and $\sigma_{\mathrm{b}}=e \mu n_{\mathrm{b}}$, eq 2 can be written as follows:

$$
\begin{aligned}
& G_{\text {total }}=\frac{e \mu \tilde{n}_{\mathrm{s}} z_{0} W}{L}+\frac{e \mu n_{\mathrm{b}}\left(D-z_{0}\right) W}{L} \\
& \quad=\frac{e \mu W}{L}\left[\widetilde{n}_{\mathrm{s}} z_{0}+n_{\mathrm{b}}\left(D-z_{0}\right)\right]=\frac{e \mu W}{L}\left[z_{0}\left(\widetilde{n}_{\mathrm{s}}-n_{\mathrm{b}}\right)\right. \\
& \left.\quad+n_{\mathrm{b}} D\right]
\end{aligned}
$$

This case is described by the situation depicted in Figure 1.
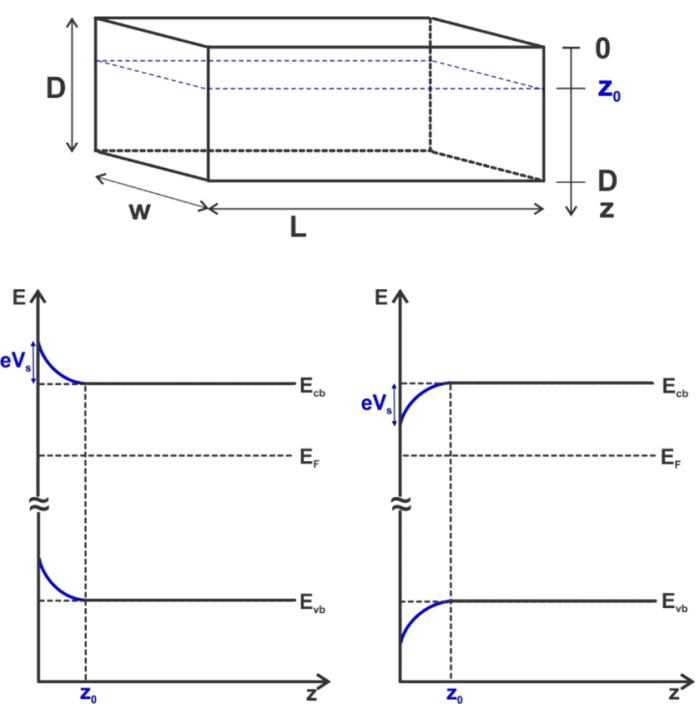

Figure 1. (top) Representation of a compact layer and its geometrical characteristics: $W$-width; $L$-length; $D$-thickness; $z_{0}$-thickness of the space charge layer; (bottom left) representation of thickness dependence of the energy bands in the semiconductor for the case of negative surface charge/formation of a depletion layer; (bottom right) representation of thickness dependence of the energy bands in the semiconductor for the case of positive surface charge/formation of an accumulation layer.

In Figure 1, $V_{\mathrm{S}}$ is defined as the difference between the electrostatic potential in the bulk and at the surface and $e V_{\mathrm{S}}$ represents the corresponding energy difference. In the case of negative surface charge, the electrons will be repelled from the surface, which means that they will need more energy to get there. This is described in the energy band representation by an upward band bending and, correspondingly, the region close to the surface will have a lower density of electrons-a surface depletion layer is formed. Its extent, $z_{0}$, depends on the surface charge and the properties of the respective SMOX. The opposite happens in the case of positive surface charge, a case in which a surface accumulation layer is formed. Detailed explanations are provided in ref 22.

Assuming the validity of the Boltzmann statistics in the whole layer, one obtains (for detailed calculations see the Supporting Information) the dependence of the overall conductance on the surface potential (band-bending) 


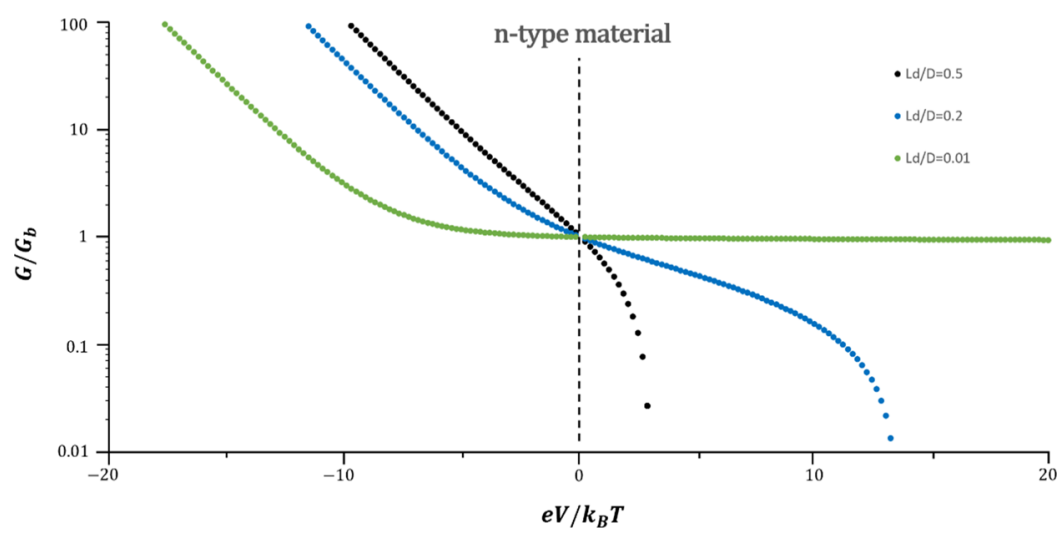

Figure 2. Normalized conduction of an n-type SMOX sensing layer as a function of surface band bending for three different values of $L_{\mathrm{D}} / D$; negative values of the band bending correspond to the formation of a surface accumulation layer, whereas positive values of the band bending correspond to the formation of a surface depletion layer.

$$
G_{\text {total }}=G_{\mathrm{b}}\left[1 \pm \sqrt{2} \frac{L_{\mathrm{D}}}{D}\left[\exp \left(-\frac{e V_{\mathrm{s}}}{k_{\mathrm{B}} T}\right)+\frac{e V_{\mathrm{s}}}{k_{\mathrm{B}} T}-1\right]^{1 / 2}\right]
$$

There are two possibilities, $V_{\mathrm{s}}>0$, which indicates the formation of a depletion layer, and $V_{s}<0$, which means the formation of an accumulation layer. In the first case, the conductance will decrease so one would have to use-in eq 4; in the second case, the conductance will increase so one would have to use + in eq 4 .

For the case of the depletion layer, $V_{\mathrm{s}}>0$ eq 4 becomes

$$
G_{\text {total }}=G_{\mathrm{b}}\left[1-\sqrt{2} \frac{L_{\mathrm{D}}}{D}\left[\exp \left(-\frac{e V_{\mathrm{s}}}{k_{\mathrm{B}} T}\right)+\frac{e V_{\mathrm{s}}}{k_{\mathrm{B}} T}-1\right]^{1 / 2}\right]
$$

In case of very small band bending when compared to the thermal energy, $e \mid V_{\mathrm{s}} \mathrm{l} / k_{\mathrm{B}} T \ll 1$, one can use a Taylor series approximation for $\exp \left(-e V_{\mathrm{s}} / k_{\mathrm{B}} T\right)$ and keep just the first three terms

$$
\begin{aligned}
& {\left[\exp \left(-\frac{e V_{\mathrm{s}}}{k_{\mathrm{B}} T}\right)+\frac{e V_{\mathrm{s}}}{k_{\mathrm{B}} T}-1\right]^{1 / 2}} \\
& \cong\left[1-\frac{e V_{\mathrm{s}}}{k_{\mathrm{B}} T}+\frac{1}{2}\left(\frac{e V_{\mathrm{s}}}{k_{\mathrm{B}} T}\right)^{2}+\ldots+\frac{e V_{\mathrm{s}}}{k_{\mathrm{B}} T}-1\right]^{1 / 2} \\
& \cong \frac{1}{\sqrt{2}}\left(\frac{e V_{\mathrm{s}}}{k_{\mathrm{B}} T}\right)
\end{aligned}
$$

Equation 5 becomes

$$
G_{\text {total }}=G_{\mathrm{b}}\left[1-\frac{L_{\mathrm{D}}}{D}\left(\frac{e V_{\mathrm{s}}}{k_{\mathrm{B}} T}\right)\right]
$$

which indicates a linear dependency on $V_{\mathrm{S}}$ around 0 .

In case of very large band bending when compared to the thermal energy, $e\left|V_{\mathrm{s}}\right| / k_{\mathrm{B}} T \gg 1$

$$
\left[\exp \left(-\frac{e V_{\mathrm{s}}}{k_{\mathrm{B}} T}\right)+\frac{e V_{\mathrm{s}}}{k_{\mathrm{B}} T}-1\right]^{1 / 2} \cong\left(\frac{e V_{\mathrm{s}}}{k_{\mathrm{B}} T}\right)^{1 / 2}
$$

Equation 5 becomes

$$
G_{\text {total }}=G_{\mathrm{b}}\left[1-\sqrt{2} \frac{L_{\mathrm{D}}}{D}\left(\frac{e V_{\mathrm{s}}}{k_{\mathrm{B}} T}\right)^{1 / 2}\right]
$$

This result suggests a linear dependence of the conductance on the square root of the band bending.

On the basis of eq 9 we can also identify the limit of validity for the formula described by eq 5 , namely,

$$
\begin{aligned}
& \sqrt{2} \frac{L_{\mathrm{D}}}{D}\left(\frac{e V_{\mathrm{s}}}{k_{\mathrm{B}} T}\right)^{1 / 2} \leq 1 \rightarrow \frac{e V_{\mathrm{s}}}{k_{\mathrm{B}} T} \leq\left(\frac{D}{\sqrt{2} L_{\mathrm{D}}}\right)^{2} \rightarrow e V_{\mathrm{s}} \leq \frac{k_{\mathrm{B}} T}{2} \\
& \left(\frac{D}{L_{\mathrm{D}}}\right)^{2}
\end{aligned}
$$

At larger values of the band bending, the depletion layer extends in the full layer and one needs a different approach.

For the case of the accumulation layer, $V_{s}<0$, eq 4 becomes

$$
G_{\text {total }}=G_{\mathrm{b}}\left[1+\sqrt{2} \frac{L_{\mathrm{D}}}{D}\left[\exp \left(-\frac{e V_{\mathrm{s}}}{k_{\mathrm{B}} T}\right)+\frac{e V_{\mathrm{s}}}{k_{\mathrm{B}} T}-1\right]^{1 / 2}\right]
$$

At low values of the band bending one obtains

$$
G_{\text {total }}=G_{\mathrm{b}}\left[1+\frac{L_{\mathrm{D}}}{D}\left(\frac{e \mid V_{\mathrm{s}}}{k_{\mathrm{B}} T}\right)\right]
$$

For large values of the band bending

$$
\left[\exp \left(-\frac{e V_{\mathrm{s}}}{k_{\mathrm{B}} T}\right)+\frac{e V_{\mathrm{s}}}{k_{\mathrm{B}} T}-1\right] \cong \exp \left(-\frac{e V_{\mathrm{s}}}{k_{\mathrm{B}} T}\right)=\exp \left(\frac{e\left|V_{\mathrm{s}}\right|}{k_{\mathrm{B}} T}\right)
$$

then eq 11 became

$$
\begin{aligned}
G_{\text {total }} & =G_{\mathrm{b}}\left[1+\sqrt{2} \frac{L_{\mathrm{D}}}{D}\left(\exp \frac{e\left|V_{\mathrm{s}}\right|}{k_{\mathrm{B}} T}\right)^{1 / 2}\right] \\
& =G_{\mathrm{b}}\left[1+\sqrt{2} \frac{L_{\mathrm{D}}}{D}\left(\exp \frac{e\left|V_{\mathrm{s}}\right|}{2 k_{\mathrm{B}} T}\right)\right]
\end{aligned}
$$

In extreme cases, $G_{\text {total }} \approx \exp \frac{e\left|V_{\mathrm{s}}\right|}{2 k_{\mathrm{B}} T}$ as in the case of a conduction mechanism dominated by the surface accumulation 
layer for porous layers, ${ }^{23}$ which indicates that the conduction taking place through the accumulation layer dominates.

Figure 2 presents the relationship between the normalized conduction of the compact layer $G / G_{\mathrm{b}}$ as a function of the band bending expressed in $k T$ units for the case of an n-type SMOX. Three cases are presented, namely, Debye length representing 1, 20 , and $50 \%$ from the total layer thickness.

In Figure 2, for the case of positive band bending and $L_{\mathrm{D}} / D=$ 0.5 , the validity of the model ends, that is,

$$
\sqrt{2} \frac{L_{\mathrm{D}}}{D}\left(\frac{e V_{\mathrm{s}}}{k_{\mathrm{B}} T}\right)^{1 / 2}=1
$$

already at $\frac{e V_{\mathrm{s}}}{k_{\mathrm{B}} T} \cong 2$. At higher values of the band bending, the depletion layer fully extends in the whole layer. This case is presented in the following section.

n-Type SMOX - Surface Effects That Do Affect the Full Layer. This situation is presented in Figure 3.
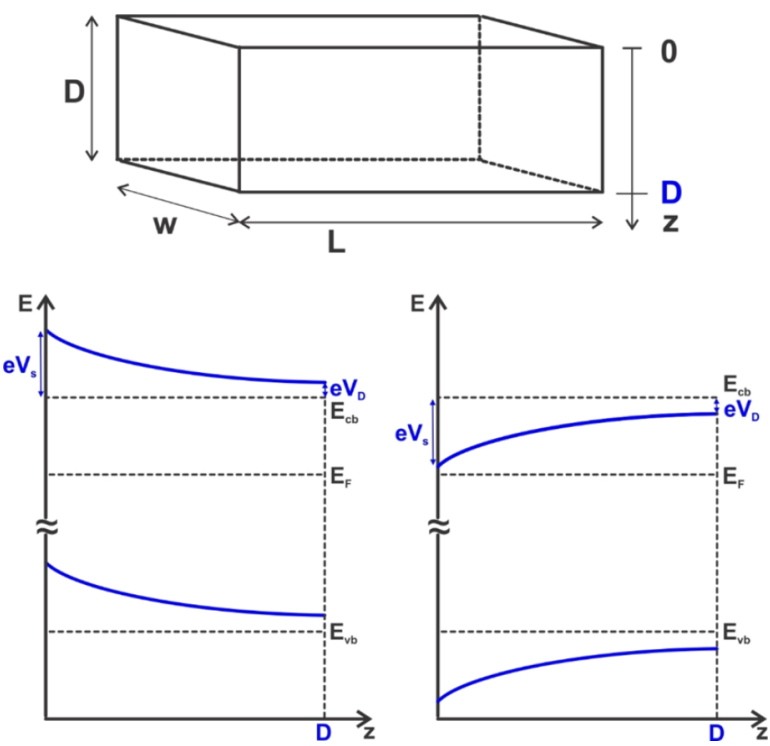

Figure 3. (top) Representation of a compact layer and its geometrical characteristics: $W$-width; $L$-length; $D$-thickness; $z_{0}$-thickness of the space charge layer; (bottom left) representation of thickness dependence of the energy bands in the semiconductor for the case of negative surface charge/formation of a depletion layer; (bottom right) representation of thickness dependence of the energy bands in the semiconductor for the case of positive surface charge/formation of an accumulation layer.

In this case, one obtains (for detailed calculations, see the Supporting Information)

$$
\begin{aligned}
G_{\text {total }}= & G_{\mathrm{b}}\left\{1 \pm \sqrt{2} \frac{L_{\mathrm{D}}}{D}\left[\exp \left(-\frac{e V_{\mathrm{s}}}{k_{\mathrm{B}} T}\right)+\frac{e V_{\mathrm{s}}}{k_{\mathrm{B}} T}-1\right]^{1 / 2}\right. \\
& \left.-\left[\exp \left(-\frac{e V_{\mathrm{D}}}{k_{\mathrm{B}} T}\right)+\frac{e V_{\mathrm{D}}}{k_{\mathrm{B}} T}-1\right]^{1 / 2}\right\}
\end{aligned}
$$

For the case of the depletion layer, $V_{\mathrm{s}}>0$, eq 16 becomes

$$
\begin{aligned}
G_{\text {total }}= & G_{\mathrm{b}}\left\{1-\sqrt{2} \frac{L_{\mathrm{D}}}{D}\left[\exp \left(-\frac{e V_{\mathrm{s}}}{k_{\mathrm{B}} T}\right)+\frac{e V_{\mathrm{s}}}{k_{\mathrm{B}} T}-1\right]^{1 / 2}\right. \\
& \left.-\left[\exp \left(-\frac{e V_{\mathrm{D}}}{k_{\mathrm{B}} T}\right)+\frac{e V_{\mathrm{D}}}{k_{\mathrm{B}} T}-1\right]^{1 / 2}\right\}
\end{aligned}
$$

For large values of the surface band bending throughout the full layer, one obtains (for detailed calculations, see the Supporting Information)

$$
G_{\text {total }} \cong G_{\mathrm{b}}\left(1-\sqrt{2} \frac{L_{\mathrm{D}}}{D} \frac{D}{\sqrt{2} L_{\mathrm{D}}}\right) \cong G_{\mathrm{b}}(1-1) \cong 0
$$

This can be interpreted as an indication that the layer will become insulating-electrical resistance $R \rightarrow \infty$.

For the case of the accumulation layer, $V_{s}<0$, eq 16 becomes

$$
\begin{aligned}
G_{\text {total }}= & G_{\mathrm{b}}\left\{1+\sqrt{2} \frac{L_{\mathrm{D}}}{D}\left[\exp \left(-\frac{e V_{\mathrm{s}}}{k_{\mathrm{B}} T}\right)+\frac{e V_{\mathrm{s}}}{k_{\mathrm{B}} T}-1\right]^{1 / 2}\right. \\
& \left.-\left[\exp \left(-\frac{e V_{\mathrm{D}}}{k_{\mathrm{B}} T}\right)+\frac{e V_{\mathrm{D}}}{k_{\mathrm{B}} T}-1\right]^{1 / 2}\right\}
\end{aligned}
$$

For large values of the surface band bending, one obtains (for detailed calculations, see the Supporting Information)

$$
G_{\text {total }} \cong G_{\mathrm{b}}\left[1+2 \exp \frac{e\left|V_{\mathrm{D}}\right|+e\left|V_{\mathrm{s}}\right|}{2 k_{\mathrm{B}} T}\right]
$$

Equation 20 suggests that the conductance of an accumulation layer fully extended throughout the full sensing layer depends exponentially on some kind of average band bending. In the case of the flat band, $e\left|V_{\mathrm{D}}\right|=e\left|V_{s}\right|$, so one obtains

$$
G_{\text {total }} \cong G_{\mathrm{b}}\left[1+2 \exp \frac{e\left|V_{\mathrm{s}}\right|}{k_{\mathrm{B}} T}\right]
$$

p-Type SMOX - Surface Effects That Do Not Affect the

Full Layer. This case is described in Figure 4.

The general form of conductance for the compact p-type SMOX layer can be written as

$$
G_{\text {total }}=G_{\mathrm{s}}+G_{\mathrm{b}} \approx \tilde{p}_{\mathrm{s}} z_{0}+p_{\mathrm{b}}\left(D-z_{0}\right)
$$

where the term $\widetilde{p}_{\mathrm{s}} z_{0}$ represents the surface contribution and the term $p_{\mathrm{b}}\left(D-z_{0}\right)$ represents the bulk contribution.

Following the same mathematical approach as in the case of ntype SMOX, one finally obtains

$$
G_{\text {total }}=G_{\mathrm{b}}\left[1 \pm \sqrt{2} \frac{L_{\mathrm{D}}}{D}\left[\exp \left(\frac{e V_{\mathrm{s}}}{k_{\mathrm{B}} T}\right)-\frac{e V_{\mathrm{s}}}{k_{\mathrm{B}} T}-1\right]^{1 / 2}\right]
$$

There are two possibilities, $V_{s}>0$, which indicates the formation of an accumulation layer, and $V_{s}<0$, which means the formation of a depletion layer. In the first case, the conductance will increase, so one would have to use + in eq 23; in the second case, the conductance will decrease; therefore, one would have to use-in eq 23.

Figure 5 presents the relationship between the normalized conduction of the compact layer $\frac{G}{G_{\mathrm{b}}}$ as a function of the band 

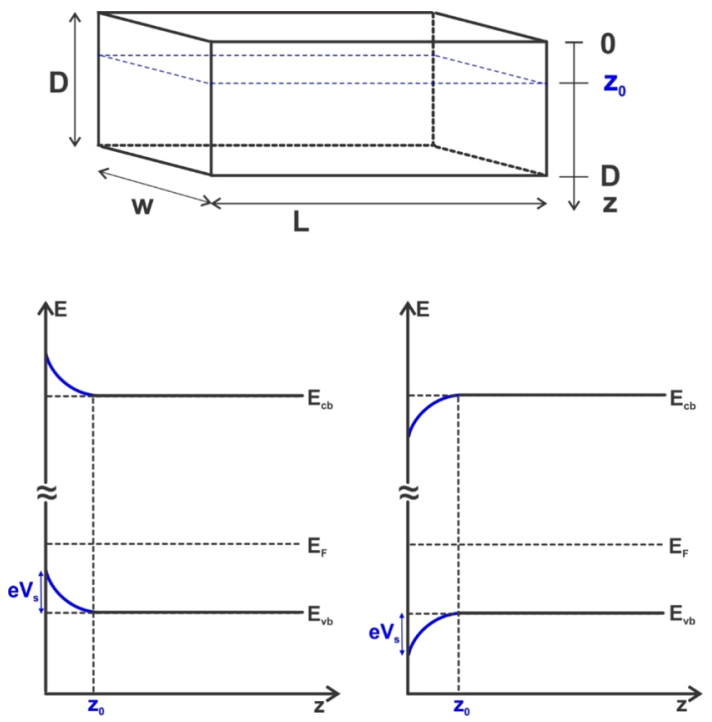

Figure 4. (top) Representation of a compact layer and its geometrical characteristics: $W$-width; $L$-length; $D$-thickness; $z_{0}$-thickness of the space charge layer; (bottom left) representation of thickness dependence of the energy bands in the semiconductor for the case of positive surface charge/formation of an accumulation layer; (bottom right) representation of thickness dependence of the energy bands in the semiconductor for the case of negative surface charge/formation of an depletion layer.

bending expressed in $k T$ units for the case of an p-type SMOX. Three cases are presented, namely, Debye length representing 1 , 20 , and $50 \%$ from the total layer thickness.

As in the case of n-type SMOX, one can obtain the limits of validity for the model, in this case for the negative band bending $-\sqrt{2} \frac{L_{\mathrm{D}}}{D}\left(-\frac{e V_{\mathrm{s}}}{k_{\mathrm{B}} T}\right)^{1 / 2}=1$, and also determine the approximations valid at very low and very large values of band bending.

For the case of the accumulation layer, $V_{s}>0$, they are

$$
G_{\text {total }}=G_{\mathrm{b}}\left[1+\frac{L_{\mathrm{D}}}{D}\left(\frac{e V_{\mathrm{s}}}{k_{\mathrm{B}} T}\right)\right]
$$

in case of very small band bending when compared to the thermal energy, $\frac{e V_{\mathrm{s}}}{k_{\mathrm{B}} T} \ll 1$ and

$$
G_{\text {total }}=G_{\mathrm{b}}\left[1+\sqrt{2} \frac{L_{\mathrm{D}}}{D} \exp \left(\frac{e V_{\mathrm{s}}}{2 k_{\mathrm{B}} T}\right)\right]
$$

in case of very large band bending when compared to the thermal energy, $\frac{e V_{\mathrm{s}}}{k_{\mathrm{B}} T} \gg 1$.

For the case of the depletion layer, $V_{\mathrm{s}}<0$, they are

$$
G_{\text {total }}=G_{\mathrm{b}}\left[1-\frac{L_{\mathrm{D}}}{D}\left(\frac{e \mid V_{\mathrm{s}}}{k_{\mathrm{B}} T}\right)\right]
$$

in case of very small band bending when compared to the thermal energy, $\frac{e\left|V_{\mathrm{s}}\right|}{k_{\mathrm{B}} T} \ll 1$ and

$$
G_{\text {total }}=G_{\mathrm{b}}\left[1-\sqrt{2} \frac{L_{\mathrm{D}}}{D}\left(\frac{e\left|V_{\mathrm{s}}\right|}{k_{\mathrm{B}} T}\right)^{1 / 2}\right]
$$

in case of very large band bending when compared to the thermal energy, $\frac{e\left|V_{\mathrm{s}}\right|}{k_{\mathrm{B}} T} \gg 1$.

p-Type SMOX - Surface Influence Extended in the Full Layer. This case is described in Figure 6.

By using the same mathematical approach like in the case of ntype SMOX one obtains.

$$
\begin{aligned}
G_{\text {total }}= & G_{\mathrm{b}}\left\{1 \pm \sqrt{2} \frac{L_{\mathrm{D}}}{D}\left[\exp \left(\frac{e V_{\mathrm{s}}}{k_{\mathrm{B}} T}\right)-\frac{e V_{\mathrm{s}}}{k_{\mathrm{B}} T}-1\right]^{1 / 2}\right. \\
& \left.-\left[\exp \left(\frac{e V_{\mathrm{D}}}{k_{\mathrm{B}} T}\right)-\frac{e V_{\mathrm{D}}}{k_{\mathrm{B}} T}-1\right]^{1 / 2}\right\}
\end{aligned}
$$

For the case of the accumulation layer, $V_{s}>0$, eq 28 becomes

$$
\begin{aligned}
G_{\text {total }}= & G_{\mathrm{b}}\left\{1+\sqrt{2} \frac{L_{\mathrm{D}}}{D}\left[\exp \left(\frac{e V_{\mathrm{s}}}{k_{\mathrm{B}} T}\right)-\frac{e V_{\mathrm{s}}}{k_{\mathrm{B}} T}-1\right]^{1 / 2}\right. \\
& \left.-\left[\exp \left(\frac{e V_{\mathrm{D}}}{k_{\mathrm{B}} T}\right)-\frac{e V_{\mathrm{D}}}{k_{\mathrm{B}} T}-1\right]^{1 / 2}\right\}
\end{aligned}
$$

For large values of the surface band bending throughout the full layer, both $\frac{e V_{\mathrm{s}}}{k_{\mathrm{B}} T}$ and $\frac{e V_{\mathrm{D}}}{k_{\mathrm{B}} T} \gg 1$, so one obtains (see the

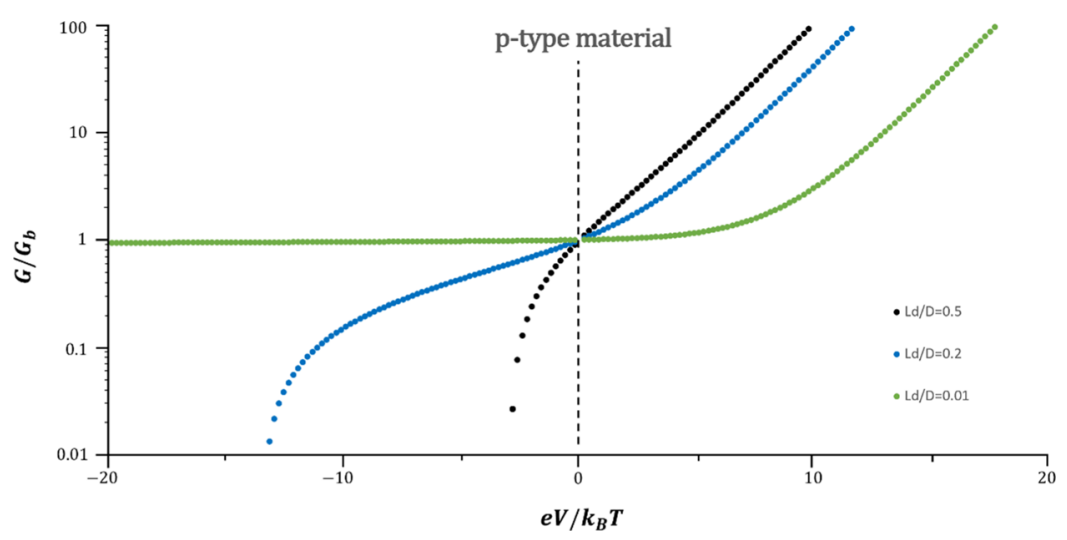

Figure 5. Normalized conduction of a p-type SMOX sensing layer as a function of surface band bending for three different values of $L_{\mathrm{D}} / D$; negative values of the band bending correspond to the formation of a surface depletion layer, whereas positive values of the band bending correspond to the formation of a surface accumulation layer. 

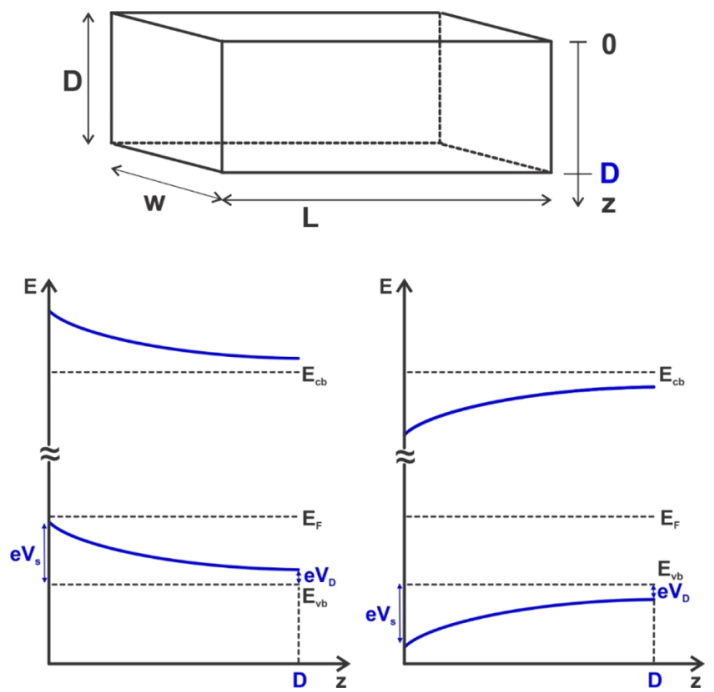

Figure 6. (top) Representation of a compact layer and its geometrical characteristics: $W$-width; $L$-length; $D$-thickness; $z_{0}$-thickness of the space charge layer; (bottom left) representation of thickness dependence of the energy bands in the semiconductor for the case of negative surface charge/formation of an accumulation layer; (bottom right) representation of thickness dependence of the energy bands in the semiconductor for the case of positive surface charge/formation of a depletion layer.

approach used in the case of n-type SMOX, eqs S34-S37 in the Supporting Information file and eq 20)

$$
G_{\text {total }} \cong G_{\mathrm{b}}\left(1+2 \exp \frac{e V_{\mathrm{D}}+e V_{\mathrm{s}}}{2 k_{\mathrm{B}} T}\right)
$$

Equation 30 suggests that the conductance of an accumulation layer fully extended throughout the full sensing layer depends exponentially on some kind of average band bending. In the case of the flat band, $e V_{\mathrm{D}}=e V_{\mathrm{s}}$, so one obtains

$$
G_{\text {total }} \cong G_{\mathrm{b}}\left[1+2 \exp \frac{e V_{\mathrm{s}}}{k_{\mathrm{B}} T}\right]
$$

For the case of the depletion layer, $V_{\mathrm{s}}<0$, eq 28 becomes

$$
\begin{aligned}
G_{\text {total }}= & G_{\mathrm{b}}\left\{1-\sqrt{2} \frac{L_{\mathrm{D}}}{D}\left[\exp \left(\frac{e V_{\mathrm{s}}}{k_{\mathrm{B}} T}\right)-\frac{e V_{\mathrm{s}}}{k_{\mathrm{B}} T}-1\right]^{1 / 2}\right. \\
& \left.-\left[\exp \left(\frac{e V_{\mathrm{D}}}{k_{\mathrm{B}} T}\right)-\frac{e V_{\mathrm{D}}}{k_{\mathrm{B}} T}-1\right]^{1 / 2}\right\}
\end{aligned}
$$

For large values of the surface band bending throughout the full layer (see the approach used in the case of n-type SMOX, eqs S26-S29), one obtains

$$
G_{\text {total }} \cong G_{\mathrm{b}}\left\{1-\sqrt{2} \frac{L_{\mathrm{D}}}{D}\left[\left(\frac{e\left|V_{\mathrm{s}}\right|}{k_{\mathrm{B}} T}\right)^{1 / 2}-\left(\frac{e\left|V_{\mathrm{D}}\right|}{k_{\mathrm{B}} T}\right)^{1 / 2}\right]\right\}
$$

which, like in the case of n-type SMOX, will tend to 0 .

\section{EXPERIMENTAL VALIDATION}

Sample Preparation and Characterization. A $280 \mathrm{~nm}$ thick, single-crystalline, unintentionally doped, rutile $\mathrm{SnO}_{2}(101)$ film was grown on a quarter of a $2^{\prime \prime}$ diameter $r$-plane sapphire substrate by plasma-assisted molecular beam epitaxy, similar to the work described in ref 32 . The rough backside of the substrate was coated by $1 \mu \mathrm{m}$ Ti to facilitate radiative heating by a heating filament behind the substrate. The substrate temperature was measured by a thermocouple placed between the heater and the substrate. Activated oxygen was provided by passing a controlled flow of molecular oxygen (6N purity) through an RF-plasma source (SPECS, PCF-RF-AN) run at a power of $300 \mathrm{~W}$. Highly pure $\mathrm{Sn}$ ( $7 \mathrm{~N}$ purity) was evaporated from a single-filament effusion cell at $1170^{\circ} \mathrm{C}$ equipped with a shutter between the cell and the substrate. The resulting flux of $\mathrm{Sn}$ corresponded to a beam equivalent pressure of $3.7 \times 10^{-7}$ Torr as measured by a nude filament ion gauge in the substrate position. Prior to growth, the substrate was exposed to an activated oxygen flux of 0.5 standard cubic centimeter per minute $(\mathrm{sccm})$ at a substrate temperature of $850{ }^{\circ} \mathrm{C}$ to improve the surface quality. After that, the substrate temperature was decreased to $750{ }^{\circ} \mathrm{C}$ and growth was initiated by opening the $\mathrm{Sn}$ shutter to grow a nucleation layer for $7 \mathrm{~min}$. The substrate temperature was subsequently ramped up to $850{ }^{\circ} \mathrm{C}$ within $100 \mathrm{~s}$ without interrupting the growth. After $26 \mathrm{~min}$ of growth, the oxygen flux was increased to $1 \mathrm{sccm}$, which improved the surface smoothness gauged by reflection high-energy electron beam diffraction (RHEED). Growth was terminated after a total growth time of $1 \mathrm{~h}$ by closing the Sn shutter, closing the oxygen flux, and cooling down the substrate in vacuum at $0.3{ }^{\circ} \mathrm{C} / \mathrm{s}$. The total film thickness is approx. $280 \mathrm{~nm}$ measured by in situ laser reflectometry during growth.

X-ray diffraction (XRD) confirmed the single crystallinity of the film with (101) out-of-plane orientation: the exclusive presence of

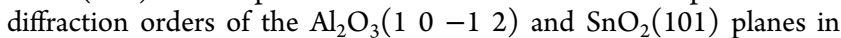
the symmetric out-of-plane $2 \Theta-\omega$ scan of the film shown in Figure $7 \mathrm{a}$
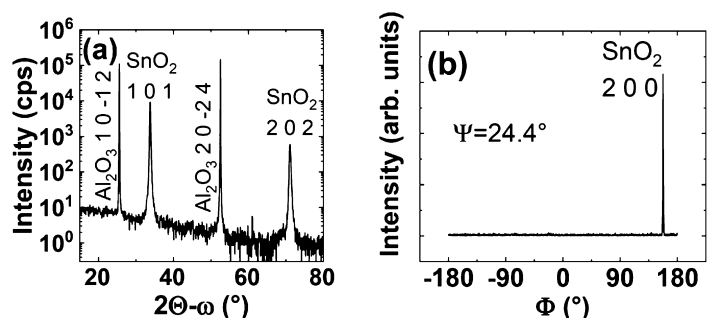

Figure 7. XRD measurements of the grown film. (a) Symmetric out-ofplane $2 \Theta-\omega$ scan. The diffraction peaks are labeled. (b) $\Phi$-scan of the skew-symmetric 200 reflection with sample tilt angle $\Psi$.

indicates a phase-pure rutile $\mathrm{SnO}_{2}(101)$ film. The presence of a single peak in the $\Phi$-scan of the skew-symmetric $\mathrm{SnO}_{2}(200)$ reflex measured by rotating the sample around its surface normal by the angle $\Phi$, shown in Figure $7 b$, indicates a single in-plane orientation without rotational domains.

The RHEED patterns shown in Figure 8 and the peak-force-tapping mode atomic force microscopy (AFM) image shown in Figure 9 indicate a smooth, unfaceted $\mathrm{SnO}_{2}$ surface.

After growth, the quarter wafer was cleaved into rectangular chips. Van-der-Pauw measurements at room temperature and in ambient air (i.e., with humidity) of a $5 \mathrm{~mm} \times 6 \mathrm{~mm}$ chip with in-contacts pressed on the film surface in the corners indicated a sheet resistance of $12 \mathrm{M} \Omega$, corresponding to a conductance of $0.083 \mu \mathrm{S}$. For the gas-response measurements, a $4 \mathrm{~mm} \times 7 \mathrm{~mm}$ chip was cleaved. Disk-shaped $20 \mathrm{~nm}$
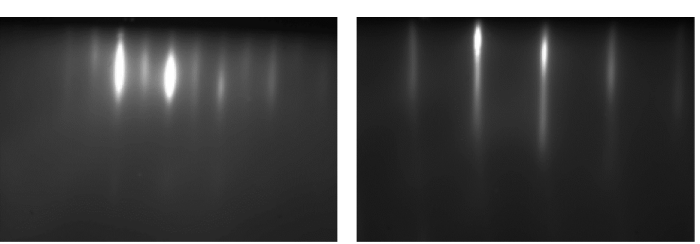

Figure 8. RHEED pattern measured after growth in two perpendicular azimuths. The patterns consisting of vertical streaks and the absence of spots indicate a smooth, crystalline surface. 


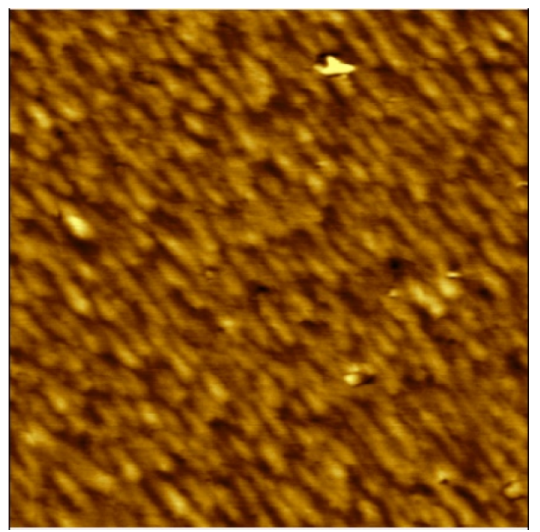

Figure 9. $2 \mu \mathrm{m} \times 2 \mu \mathrm{m}$ AFM image of the film surface. The height scale (black-to-white) is $3 \mathrm{~nm}$.

$\mathrm{Ti} / 100 \mathrm{~nm} \mathrm{Au}$ contacts with $0.5 \mathrm{~mm}$ diameter were deposited by electron-beam evaporation through a shadow mask in a $2 \mathrm{~mm} \times 2 \mathrm{~mm}$ square arrangement next to a short edge of the chip. This metallization provides an ohmic contact to $\mathrm{SnO}_{2}{ }^{28}$

Finite element calculations using the FreeFem++ software $^{33}$ were performed to relate the two-point resistance measured for the chosen contact arrangement to the sheet resistance following the approach of ${ }^{27}$ as shown in Figure 10: in the calculations for the chosen sample and
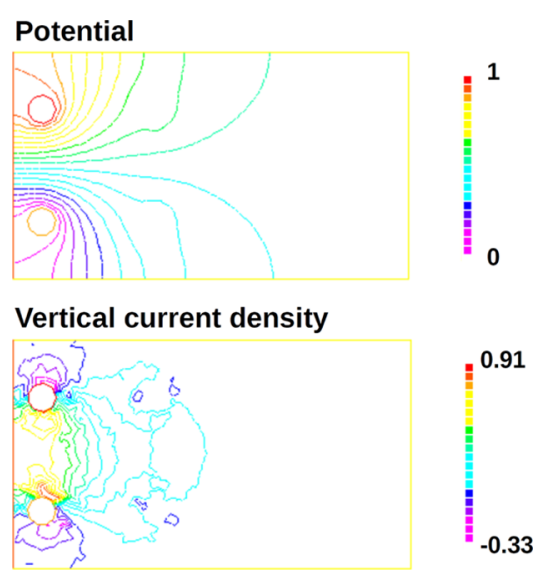

Figure 10. Finite element calculations of the two-point resistance for the chosen contact arrangement. The potential distribution and the distribution of the vertical current density component are shown.

contact geometry, potentials of 0 and $1 \mathrm{~V}$ were applied to the top and bottom contacts, respectively, and the consequent potential distribution was calculated assuming an isotropic sheet resistance of $1 \Omega$. After that, the vertical current density distribution was calculated and integrated along a horizontal line along the sample center to determine the total current between the two contacts. The ratio of the potential difference (of $1 \mathrm{~V}$ ) and the resulting current corresponds to the twopoint resistance. Assuming a negligible contact resistance, the measured two-point resistance $R_{2 p}$ corresponds to 0.97 times the sheet resistance $R_{\mathrm{S}}\left(R_{2 \mathrm{p}}=0.97 \times R_{\mathrm{S}}\right)$, which can be expressed by a geometry factor of $\mathrm{W} /$ $L=1 / 0.97=1.03$.

The chip was subsequently glued with fast-drying silver paint on a ceramics carrier and two of the ohmic contacts were wire-bonded to the contact lines on the carrier to enable in-operando resistance measurements. Figure 11 shows a photograph of the processed sample.

Operando Work Function/dc Resistance Measurements. Two-point dc resistance measurements combined with simultaneously performed work function changes' measurements were done at the regular operation temperature of SMOX sensors. ${ }^{12}$ Work function changes were measured with the Kelvin Probe technique, which is a

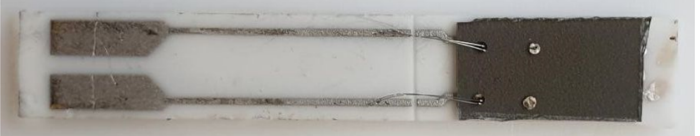

Figure 11. Photograph of the sample chip with ohmic contacts mounted on a ceramics carrier.

noncontact, nondestructive method that uses a vibrating reference electrode and measures the changes of the contact potential difference (CPD) between the sample and the electrode. Variations in the CPD induced by the changes in the gas atmosphere represent relative work function variations of the sample. ${ }^{24}$ The sensor, operated at $300{ }^{\circ} \mathrm{C}$, which is the normal operation temperature for $\mathrm{SnO}_{2}$-based gas sensors, was exposed to

- 5, 10, 20, 50, and 100 ppm of $\mathrm{H}_{2}$ and $\mathrm{CO}$ and 25, 50, 100, 250, 500,1000 , and $2500 \mathrm{ppm}$ of $\mathrm{O}_{2}$ in a background of $\mathrm{N}_{2}$;

- 5, 10, 20, 50, and 100 ppm of $\mathrm{CO}$ and 2, 5, 10, and 20 ppm of $\mathrm{NO}_{2}$ in a background of synthetic air.

The total gas flow was $400 \mathrm{sccm}$. The reason for the choice of the different compositions of the ambient atmosphere of the sensor was to ensure a significant variation of both positive and negative band bending.

Because the measurements were done in a very dry atmosphere, we assume that the electron affinity remains constant during the whole process. ${ }^{12}$ Also, at the working temperature of $300{ }^{\circ} \mathrm{C}$, we assume that gas interactions are limited to the surface. Taking this into account, the changes in CPD upon different gas exposures can be related to work function changes as

$$
\Delta \mathrm{CPD}=-\Delta \varnothing=-e \Delta V_{\mathrm{s}}
$$

A detailed description of work function and Kelvin Probe working principles can be found in ref 24 .

The dependence of the sample conductance on band bending is shown in Figure 12. In order to obtain a good fit, the experimental points were allowed to move along the direction of the band bending, which means that we took into account the possibility that in $\mathrm{N}_{2}$ there is a downward or upward band bending. The best fit to the model was obtained by considering an upward band bending of $0.22 \mathrm{eV}, G_{\mathrm{b}}=11$ $\mu \mathrm{S}$ and $L_{\mathrm{D}} / D=0.115$ as values for the fit parameters. Using these data, we obtained $L_{\mathrm{D}}=32 \mathrm{~nm}$ for the Debye Length, and $n_{\mathrm{b}}=3.15 \times 10^{22} \mathrm{~m}^{-3}$ for the bulk electron concentration-in the calculations we used $\epsilon_{r}=12$ as an average value. ${ }^{25}$

Consequently, there is an effect, not related to oxygen adsorption, that leads to an upward band bending in the absence of an oxidizing atmosphere (in pure $\mathrm{N}_{2}$ ). A similar situation was found in ref 26 for undoped polycrystalline $\mathrm{SnO}_{2}$ where this behavior was attributed to a high oxygen vacancy concentration where some $\mathrm{Sn}^{+4}$ atoms at the surface change to $\mathrm{Sn}^{+2}$ and acted as surface electron acceptors. This intrinsic band bending has an influence on the electrical behavior as it affects the concentration of surface species and particularly, see ref 26 , increased the sensitivity in comparison with a sample that showed no intrinsic band bending under the same conditions. Using the formula for bulk conductance $G_{\mathrm{b}}=\frac{e \mu W n_{\mathrm{b}} D}{L}$ and the geometrical factor $W / L=$ 1.03 of our contact geometry, one obtains an electron mobility of $\mu=75$ $\mathrm{cm}^{2} / \mathrm{V} \mathrm{s}$, which is in good agreement with the (phonon-limited) electron Hall mobility of $60 \mathrm{~cm}^{2} / \mathrm{V} \mathrm{s}$ measured in single-crystalline $\mathrm{SnO}_{2}$ at $300{ }^{\circ} \mathrm{C}$. $^{31}$

For the calculated bulk electron concentration, the distance between the bottom of the conduction band and the Fermi level can be estimated from

$$
\left(E_{\mathrm{C}}-E_{\mathrm{F}}\right)=\frac{k_{\mathrm{B}} T}{e} \ln \left[\frac{N_{\mathrm{C}}}{n_{\mathrm{b}}}\right]
$$

Considering that the effective density of states of $N_{C}=9.5 \times 10^{24} \mathrm{~m}^{-3}$ based on a density-of-states effective mass of 0.275 times the free 


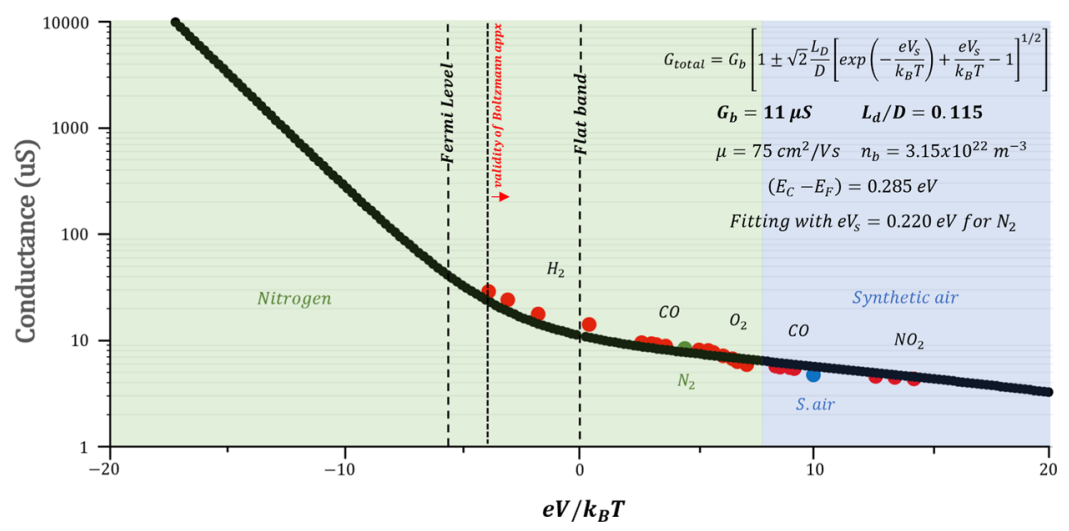

Figure 12. Conductance against relative band bending changes. The green and blue area represents the carrier gas present. Allowing experimental points to move along the $x$ direction, a flat band can be found for the first $\mathrm{H}_{2}$ concentration step and, for pure $\mathrm{N}_{2}$, a band bending of $0.22 \mathrm{eV}$. From the fitting parameters $G_{\mathrm{b}}$ and $L_{\mathrm{D}} / D$, the electronic bulk concentration $n_{\mathrm{b}}=3.15 \times 10^{22} \mathrm{~m}^{-3}$ and the mobility $\mu=75 \mathrm{~cm}^{2} / \mathrm{V} \mathrm{s}$ were calculated.

electron mass ${ }^{30}$ and a temperature of $573 \mathrm{~K}$, one finds $\left(E_{\mathrm{C}}-E_{\mathrm{F}}\right)=$ $0.285 \mathrm{eV}$.

The black dots represent the full analytical solution of eq 4 for energies between -1 and $1 \mathrm{eV}$; for values of band bending exceeding/ on the left of the "validity of Boltzmann appx", it is expected that the experimental points will not sit on the black curve. To address this possible issue, numerical models need to be developed. Luckily, in this case, all experimental data points are within the validity range of the Boltzmann approximation.

\section{CONCLUSIONS}

We developed a theoretical model that correlates the conductance of compact $n$ - and p-type SMOX single-crystalline films to the changes in the surface electrostatic potential in the nondegenerate limit. We were also able to apply it to the interpretation of experimental data obtained for an epitaxial $\mathrm{SnO}_{2}$ (101) sensing layer, operated at $300{ }^{\circ} \mathrm{C}$ and at normal pressure-the investigations were simultaneously performed work function changes and dc conductance measurements. The insights that were gained demonstrate that it is possible to acquire fundamental knowledge that is needed for the basic understanding of gas-sensing with SMOX. Specifically, the results presented here demonstrate that it is possible to gain significant insights into the "transduction function" of $\mathrm{SnO}_{2}$ epitaxial layers by using combined work function/dc resistance measurements. The next steps will be to use them, in combination with other operando investigation techniques, to unravel fundamental questions such the dependence of the "receptor function" on the crystallographic surface plane. We also intend to extend our studies to other n- and p-type SMOX single-crystalline films.

\section{ASSOCIATED CONTENT}

\section{S Supporting Information}

The Supporting Information is available free of charge on the ACS Publications website at DOI: 10.1021/acssensors.9b01018.

Full mathematical derivations (PDF)

\section{AUTHOR INFORMATION}

\section{Corresponding Author}

*E-mail: nb@ipc.uni-tuebingen.de.

ORCID $\odot$

Nicolae Barsan: 0000-0001-6718-9889

\section{Notes}

The authors declare no competing financial interest.

\section{ACKNOWLEDGMENTS}

C.E.S. and A.S. would like to thank the Romanian National Authority for Scientific Research for funding through the Core Program PN19-03 (contract no. 21 N/08.02.2019) and project PN-III-P1-1.1-MC-2017-1917/2017. The work of F.S. was supported by a Georg Forster Fellowship from the Alexander von Humboldt Foundation. Also, Federico would like to thank Nicolas Tibaldi for assistance with the graphical abstract. Work at Paul-Drude-Institut (PDI) was performed in the framework of GraFOx, a Leibniz-ScienceCampus partially funded by the Leibniz association. We thank Hans-Peter Schönherr (PDI) for technical MBE support as well as Walid Anders (PDI) and Angela Riedel (PDI) for sample processing.

\section{REFERENCES}

(1) Heiland, G. Zum Einfluß von adsorbiertem Sauerstoff auf die elektrische Leitfähigkeit von Zinkoxydkristallen. Z. Phys. 1954, 138, 459-464.

(2) Heiland, G. Zum Einfluß von Wasserstoff auf die elektrische Leitfähigkeit an der Oberfläche von Zinkoxydkristallen. Z. Phys. 1957, $148,15-27$.

(3) Seiyama, T.; Kato, A.; Fujiishi, K.; Nagatani, M. A New Detector for Gaseous Components Using Semiconductive Thin Films. Anal. Chem. 1962, 34, 1502-1503.

(4) Taguchi, N. Gas-Detecting Device. U.S. Patent 3,631,436A, 1-2 Ikedauemachi Nagata-ku, Kobe, Japan. Patented, Dec 28, 1971.

(5) Watson, J.; Ihokura, K.; Coles, G. S. V. The tin dioxide gas sensor. Meas. Sci. Technol. 1993, 4, 711-719.

(6) Barsan, N.; Gauglitz, G.; Oprea, A.; Ostertag, E.; Proll, G.; Rebner, K.; Schierbaum, K.; Schleifenbaum, F.; Weimar, U. Chemical and Biochemical Sensors, 1. Fundamentals. Ullmann's Encyclopedia of Industrial Chemistry; Wiley-VCH Verlag $\mathrm{GmbH} \& \mathrm{Co}$, 2016; pp 1-81

(7) Simon, I.; Bârsan, N.; Bauer, M.; Weimar, U. Micromachined metal oxide gas sensors: Opportunities to improve sensor performance. Sens. Actuators, B 2001, 73, 1-26.

(8) Briand, D.; Courbat, J. Micromachined semiconductor gas sensors. In Semiconductor Gas Sensors; Jaaniso, R., Tan, O. K., Eds., Woodhead Publishing Series in Electronics and Optical Materials Number 38; Woodhead Publishing Limited, 2013; ISBN 978-0-85709236-6, pp 220-260.

(9) Rüffer, D.; Hoehne, F.; Bühler, J. New Digital Metal-Oxide (MOx) Sensor Platform. Sensors 2018, 18, 1052.

(10) Yamazoe, N. New approaches for improving semiconductor gas sensors. Sens. Actuators, B 1991, 5, 7-19. 
(11) Bârsan, N.; Huebner, M.; Weimar, U. Conduction meachanism in semiconducting metal oxides sensing films: impact on transduction. In Semiconductor Gas Sensors; Jaaniso, R., Tan, O. K., Eds.; Series in Electronics and Optical Materials; Woodhead Publishing, 2013; Vol. 38, pp 35-63.

(12) Bârsan, N.; Hübner, M.; Weimar, U. Conduction mechanisms in $\mathrm{SnO} 2$ based polycrystalline thick film gas sensors exposed to $\mathrm{CO}$ and $\mathrm{H} 2$ in different oxygen backgrounds. Sens. Actuators, B 2011, 157, 510517.

(13) Hübner, M.; Bârsan, N.; Weimar, U. Influences of Al, Pd and Pt additives on the conduction mechanism as well as the surface and bulk properties of $\mathrm{SnO} 2$ based polycrystalline thick film gas sensors”. Sens. Actuators, B 2012, 171-172, 172-180.

(14) Hübner, M.; Koziej, D.; Grunwaldt, J. D.; Weimar, U.; Barsan, N. $\mathrm{An} \mathrm{Au}$ clusters related spill-over sensitization mechanism in SnO2based gas sensors identified by operando HERFD-XAS, work function changes, DC resistance and catalytic conversion studies. Phys. Chem. Chem. Phys. 2012, 14, 13249-13254.

(15) Choi, K.-I.; Hübner, M.; Haensch, A.; Kim, H.-J.; Weimar, U.; Barsan, N.; Lee, J.-H. Ambivalent effect of Ni loading on gas sensing performance in $\mathrm{SnO} 2$ based gas sensor. Sens. Actuators, B 2013, 183, 401-410.

(16) Rebholz, J.; Bonanati, P.; Jaeschke, C.; Hübner, M.; Mädler, L.; Weimar, U.; Barsan, N. Conduction mechanism in undoped and antimony doped $\mathrm{SnO} 2$ based FSP gas sensors. Sens. Actuators, B 2013, 188, 631-636.

(17) Barsan, N.; Rebholz, J.; Weimar, U. Conduction mechanism switch for $\mathrm{SnO} 2$ based sensors during operation in application relevant conditions; implications for modeling of sensing. Sens. Actuators, B 2015, 207, 455-459.

(18) Barsan, N.; Weimar, U. Conduction Model of Metal Oxide Gas Sensors. J. Electroceram. 2001, 7, 143-167.

(19) Rombach, J.; Papadogianni, A.; Mischo, M.; Cimalla, V.; Kirste, L.; Ambacher, O.; Berthold, T.; Krischok, S.; Himmerlich, M.; Selve, S.; Bierwagen, $\mathrm{O}$. The role of surface electron accumulation and bulk doping for gas-sensing explored with single-crystalline $\mathrm{In} 2 \mathrm{O} 3$ thin films. Sens. Actuators, B 2016, 236, 909.

(20) Berthold, T.; Katzer, S.; Rombach, J.; Krischok, S.; Bierwagen, O.; Himmerlich, M. Towards Understanding the Cross-Sensitivity of In2O3 Based Ozone Sensors: Effects of O3, O2 and $\mathrm{H} 2 \mathrm{O}$ Adsorption at In2O3(111) Surfaces. Phys. Status Solidi 2018, 255, 1700324.

(21) Wicker, S.; Guiltat, M.; Weimar, U.; Hemeryck, A.; Barsan, N. Ambient Humidity Influence on CO Detection with $\mathrm{SnO} 2$ Gas Sensing Materials. A Combined DRIFTS/DFT Investigation. J. Phys. Chem. C 2017, 121, 25064-25073.

(22) Morrison, S. R. The Chemical Physics of Surfaces; Plenum: New York, 1977; p 36, ISBN 0-306-30960-2, Sec. 2.2.

(23) Barsan, N.; Simion, C.; Heine, T.; Pokhrel, S.; Weimar, U. Modeling of sensing and transduction for p-type semiconducting metal oxide based gas sensors. J. Electroceram. 2010, 25, 11-19.

(24) Oprea, A.; Bârsan, N.; Weimar, U. Work function changes in gas sensitive materials: Fundamentals and applications. Sens. Actuators, $B$ 2009, 142, 470-493.

(25) Batzill, M.; Diebold, U. The surface and materials science of tin oxide. Prog. Surf. Sci. 2005, 79, 47.

(26) Rebholz, J.; Dee, C.; Weimar, U.; Barsan, N. A self-doping surface effect and its influence on the sensor performance of undoped $\mathrm{SnO} 2$ based gas sensors. Procedia Eng. 2015, 120, 83-87.

(27) Bierwagen, O.; Pomraenke, R.; Eilers, S.; Masselink, W. T. Mobility and carrier density in materials with anisotropic conductivity revealed by van der Pauw measurements. Phys. Rev. B: Condens. Matter Mater. Phys. 2004, 70, 1-6.

(28) Bierwagen, O.; White, M. E.; Tsai, M. Y.; Nagata, T.; Speck, J. S. Non-alloyed schottky and ohmic contacts to as-grown and oxygenplasma treated n-type $\mathrm{SnO} 2(110)$ and (101) thin films. Appl. Phys. Express 2009, 2, 106502.

(29) Budde, M.; Tschammer, C.; Franz, P.; Feldl, J.; Ramsteiner, M.; Goldhahn, R.; Bierwagen, O. Structural, optical, and electrical properties of unintentionally doped $\mathrm{NiO}$ layers grown on $\mathrm{MgO}$ by plasma-assisted molecular beam epitaxy. J. Appl. Phys. 2018, 123, 195301.

(30) Button, K. J.; Fonstad, C. G.; Dreybrodt, W. Determination of the Electron Masses in Stannic Oxide by Submillimiter Cyclotron Resonance. Phys. Rev. B: Condens. Matter Mater. Phys. 1971, 4, $4539-4542$

(31) Fonstad, C. G.; Rediker, R. H. Electrical properties of highquality stannic oxide crystals. J. Appl. Phys. 1971, 42, 2911-2918.

(32) White, M. E.; Tsai, M. Y.; Wu, F.; Speck, J. S. Plasma-assisted molecular beam epitaxy and characterization of $\mathrm{SnO} 2(101)$ on r-plane sapphire. J. Vac. Sci. Technol. A 2008, 26, 1300-1307.

(33) Ohtsuka, K.; Pironneau, O.; Bernardi, D.; Hecht, F. Freefem+ a Finite Element Software to Handle Several Meshes, http://www3. freefem.org/ff++/freefem/index.html, 1999. 